\title{
NOTE ON MULTIPLY-INFINITE SERIES
}

I. M. SHEFFER

Let $\sum a_{(i)}$ be a $k$-tuply infinite series, where the subscript ( $i$ ) stands for the set of $k$ indices $i_{1}, \cdots, i_{k}$ and where for each $s=1, \cdots, k$ the index $i_{s}$ ranges from 0 to $\infty$. If $\sum b_{(i)}$ is a second such series we can determine from them a third series $\sum c_{(i)}$ called the Cauchy-product series, defined by

$$
c_{(i)}=\sum a_{(j)} b_{(n)},
$$

where the sum is over all indices for which, simultaneously,

$$
j_{s}+n_{s}=i_{s}, \quad s=1, \cdots, k .
$$

Series $\sum c_{(i)}$ is obtained by formally multiplying the two power series $\sum a_{(i)} t_{1}^{\alpha} \cdots t_{k}^{\lambda}, \sum b_{(i)} t_{1}^{\alpha} \cdots t_{k}^{\lambda}\left(\alpha \equiv i_{1}, \cdots, \lambda \equiv i_{k}\right)$, then setting $t_{s}=1$, $s=1, \cdots, k$.

Given that $\sum a_{(i)}, \sum b_{(i)}$ converge to $A, B$ respectively, it is natural to ask if $\sum c_{(i)}$ possesses the Cauchy-product property, that is, if it converges and to the sum $C=A B$; and if this is not always true, under what further conditions it will be true. It is the purpose of this note to give an answer to this question. All convergence is to be in the sense of Pringsheim (except in the concluding remark).

For simply-infinite series (hence also for multiple series) mere convergence of the series $\sum a_{(i)}, \sum b_{(i)}$ is known to be insufficient to insure that $\sum c_{(i)}$ will converge to the right sum. A theorem of Mertens states however that if both given series converge, one of them absolutely, then the Cauchy-product property holds.

Like a number of other properties of simple series, the Mertens theorem does not go over unrestrictedly to multiple series that are Pringsheim convergent. We shall show this by an example, after which we shall find suitable restrictions that will restore the Cauchyproduct property.

Example. Let $\sum a_{i j}, \sum b_{i j}$ be the following double series: $\sum a_{i j}$ is the absolutely convergent series whose first column has the elements

$$
1, \frac{1}{2}, \frac{1}{2^{2}}, \ldots, \frac{1}{2^{n}}, \ldots,
$$

while all other terms are zero; $\sum b_{i j}$ is the convergent series whose

Presented to the Society, April 27, 1946; received by the editors June 5, 1946. 
first two rows are

$$
\begin{array}{r}
0 !+1 !+\cdots+n !+\cdots \\
-0 !-1 !-\cdots-n !-\cdots
\end{array}
$$

all other terms being zero. It is readily ascertained that in the series $\sum c_{i j}$,

$$
c_{0 j}=j ! ; \quad c_{i j}=-j ! / 2^{i}, \quad i>0 ;
$$

and that

$$
R_{p q}^{C}=\frac{1}{2^{p}}(0 !+1 !+\cdots+q !)
$$

where $R_{p q}^{C}$ designates the $(p, q)$ th rectangular partial sum in the $c$-series:

$$
R_{p q}^{c} \equiv \sum c_{i j} \quad(0 \leqq i \leqq p, 0 \leqq j \leqq q) .
$$

Relation (3) shows that $R_{p q}^{C}$ does not have a limit as $p, q \rightarrow \infty$; so series $\sum c_{i j}$ does not even converge.

Let $\left\{R_{(i)}^{B}\right\}$ denote the set of rectangular partial sums for the convergent series $\sum b_{(i)}$ :

$$
R_{(i)}^{B} \equiv \sum b_{(j)} \quad\left(0 \leqq j_{s} \leqq i_{s}, s=1,2, \cdots, k\right) .
$$

The boundedness of this set is what we need to validate the Cauchyproduct property. This is shown by the theorems that follow.

THEOREM 1. Let $A=\sum a_{(i)}, B=\sum b_{(i)}$ be convergent, the former convergence being absolute. If the set $\left\{R_{(i)}^{B}\right\}$ is bounded, then $\sum c_{(i)}$ converges to the sum $C=A B$.

For convenience in notation we give the proof for double series $(k=2)$, and it will be seen to carry over to the general case of $k$-tuple series. Define $A^{\prime}, M$ by

$$
\begin{aligned}
A^{\prime}=\sum\left|a_{i j}\right| & (i, j=0,1, \cdots), \\
M=\text { l.u.b. }\left|R_{i j}\right| & (i, j=0,1, \cdots) .
\end{aligned}
$$

Let $\epsilon>0$ be given. There exist indices $a, b$ such that for all $t \geqq a$, $w \geqq b$,

$$
\left|R_{t, w}^{A}-A\right|<\epsilon
$$

and also such that

$$
\sum^{*}\left|a_{i j}\right|<\epsilon
$$


where $\sum^{*}$ is the sum of the absolute values of all terms of $\sum a_{i i}$ that are exterior to $R_{a b}^{A}$.

Now we have

$$
\begin{aligned}
R_{t w}^{c} & =\sum a_{p q} b_{r s} & (0 \leqq p+r \leqq t, 0 \leqq q+s \leqq w) \\
& =\sum a_{p q} R_{t-p, w-q}^{B} & (0 \leqq p \leqq t, 0 \leqq q \leqq w) ;
\end{aligned}
$$

and this last sum can be split into two:

$$
R_{t w}^{c}=\sum^{\prime}+\sum^{\prime \prime}
$$

where $\sum^{\prime}$ is summed over the set $(0 \leqq p \leqq a, 0 \leqq q \leqq b)$, and $\sum^{\prime \prime}$ over $(0 \leqq p \leqq t, 0 \leqq q \leqq w)$ with the additional restriction that in every term either $p>a$ or $q>b$. From (7) and (9), $\left|\sum^{\prime \prime}\right| \leqq M \epsilon$, so

$$
\left|R_{t w}^{c}-\sum^{\prime}\right| \leqq M \epsilon \quad(t \geqq a, w \geqq b) .
$$

Let $\lambda_{p q}$ be defined by

$$
B=R_{p q}^{B}+\lambda_{p q}
$$

then indices $u_{1}, v_{1}$ exist so that

$$
\left|\lambda_{p q}\right|<\epsilon \quad\left(p \geqq u_{1}, q \geqq v_{1}\right) .
$$

Hence if $t \geqq u=a+u_{1}, w \geqq v=b+v_{1}$, then

$R_{t w}^{C}-\sum^{\prime}=R_{t w}^{C}-B R_{a b}^{A}+\sum a_{p q} \lambda_{t-p, w-q} \quad(0 \leqq p \leqq a, 0 \leqq q \leqq b) ;$ so from (12),

$$
\left|R_{t w}^{c}-B R_{a b}^{A}\right| \leqq\left(M+A^{\prime}\right) \epsilon \quad(t \geqq u, w \geqq v) .
$$

Combining this with (8) we obtain

$$
\left|R_{t w}^{C}-A B\right| \leqq\left(M+A^{\prime}+|B|\right) \epsilon \quad(t \geqq u, w \geqq v) ;
$$

so series $\sum c_{i j}$ converges to $C=A B$, as was to be shown.

It may be asked if the condition of boundedness of the set $\left\{R_{(i)}^{B}\right\}$ is the mildest that will achieve the desired purpose. That this is in a sense so is shown by the following theorem.

Theorem 2. Let $B=\sum b_{(s)}$ be convergent. If set $\left\{R_{(i)}^{B}\right\}$ is unbounded, then there exists an absolutely convergent series $\sum a_{(i)}$ for which the Cauchy-product property fails to hold; in fact, for which series $\sum c_{(i)}$ does not even converge.

We first give the proof for $k=2$, after which we shall outline the corresponding proof in the general $k$-tuple case. For a given $\epsilon>0$, indices $(p, q)$ exist so that $\left|B-R_{\mathfrak{v}}^{B}\right|<\epsilon$ for all $i>p, j>q$. Hence, since $\left\{R_{\mathfrak{v}}^{B}\right\}$ 
is unbounded, either there is a fixed $u$ such that $\left\{R_{u, j}^{B}\right\}(j=0,1, \cdots)$ is unbounded, or there is a fixed $v$ such that $\left\{R_{i_{0}}^{B}\right\}(i=0,1, \cdots)$ is unbounded. It is no restriction to suppose that $\left\{R_{u j}^{B}\right\}$ is unbounded; and moreover, that $u$ is the largest integer having this property. Then for a suitably chosen sequence of increasing integers $j_{n}(n=1,2, \cdots)$,

$$
\lim _{n \rightarrow \infty}\left|R_{u, j(n)}^{B}\right|=\infty \quad\left(j(n) \equiv j_{n}\right) .
$$

We now define the absolutely convergent series $\sum a_{i j}$ as follows:

$$
\begin{array}{rlrl}
a_{i j}=0, j=1,2, \cdots ; & a_{20}=0, i \neq r(u+1), & r=0,1, \cdots ; \\
a_{r(u+1), 0}=(r+1)^{-2}, & r=0,1, \cdots .
\end{array}
$$

From (10) we readily find that for $s=1,2, \cdots$,

$$
\begin{aligned}
R_{s u+s-1, j(n)}^{C}= & \frac{1}{1^{2}} R_{s u+s-1, j(n)}^{B}+\frac{1}{2^{2}} R_{(s-1) u+s-2, j(n)}^{B}+\cdots \\
& +\frac{1}{s^{2}} R_{u, j(n)}^{B} .
\end{aligned}
$$

Suppose series $\sum c_{i j}$ converges, say to $C$. Then indices $\left(p^{\prime}, g^{\prime}\right)$ exist so that $\left|C-R_{i j}^{C}\right|<\epsilon\left(i>p^{\prime}, j>q^{\prime}\right)$. Let $s$ have a fixed value for which $s u+s-1>p^{\prime}$, and choose $N$ so that $j_{n}>q^{\prime}$ for all $n>N$. Then

$$
\left|R_{s u+s-1, j(n)}^{C}\right|<|C|+\epsilon \quad\left(j(n) \equiv j_{n} ; n>N\right) .
$$

Also, there exist constants $M_{2}, \cdots, M_{s}$ such that

$$
\left|R_{(s-m) u+8-m-1, j(n)}^{B}\right|<M_{s-m} \quad(n>N ; m=0,1, \cdots, s-2),
$$

as is seen from the maximal property of the integer $u$. Hence from (19),

$$
\left|R_{u, j(n)}^{B}\right|<s^{2}\left\{|C|+\epsilon+M_{2}+\cdots+M_{s}\right\},
$$

for all $n>N$. As this contradicts (17), it is clear that series $\sum c_{i j}$ does not converge.

Now consider the general ( $k$-tuple) case. Given $\epsilon>0$, there correspond indices $\left(p \equiv\left(p_{1}, \cdots, p_{k}\right)\right.$ so that $\left|B-R_{(i)}^{B}\right|<\epsilon$ for all $(i)>(p)$. There is therefore an index, that we may take to be $i_{1}$, such that for $i_{i}=\alpha$ (a certain integer), the set $\left\{R_{\alpha, i(2, \cdots, k)}^{B}\right\}$ is unbounded, where $i(2, \cdots, k) \equiv i_{2}, \cdots, i_{k}$. It follows that there is an integer $r$, in the range $1 \leqq r<k$, with the following properties: (i) When certain $r$ indices, which it is no restriction to take as $i_{1}, \cdots, i_{r}$, take on certain fixed values $i_{1}=\alpha, \cdots, i_{r}=\lambda$, while the remaining indices $i_{r+1}, \cdots, i_{k}$ remain arbitrary, the set $\left\{R_{\alpha, \cdots, \lambda, i(r+1, \cdots, k)}^{B}\right\}$ is unbounded. Here 
$i(r+1, \cdots, k) \equiv i_{r+1}, \cdots, i_{k}$. (ii) The integer $r$ is maximal, that is, no larger integer has property (i).

In consequence of (ii), we can assert that it is possible to choose an infinite sequence of sets $i_{r+1}, \cdots, i_{k}$ :

$$
\begin{array}{r}
\left(i_{r+1}, \cdots, i_{k}\right)_{n} \equiv i(r+1, \cdots, k)_{n} \equiv i(r+1, \cdots, k ; n), \\
n=1,2, \cdots,
\end{array}
$$

with $\lim \left(i_{s}\right)_{n}=\infty$ as $n \rightarrow \infty$ for each $s=r+1, \cdots, k$, for which

$$
\lim _{n \rightarrow \infty}\left|R_{\alpha, \cdots, \lambda, i(r+1, \cdots, k ; n)}^{B}\right|=\infty .
$$

Choose the integer $t$ large enough so that the following $r$ inequalities hold: $t \alpha+t-1>p_{1}, \cdots, t \lambda+t-1>p_{r}$; and consider the sets

$$
\left\{R_{q \alpha+q-1, \cdots, q \lambda+q-1, i(r+1, \cdots, k ; n)}^{B}\right\}, \quad q=1,2, \cdots .
$$

The set for which $q=1$ is unbounded, we know; and the set for each $q \geqq t$ is bounded, since then the $s$ th index exceeds $p_{s}$ for $s=1, \cdots, k$ (at least for $n$ sufficiently large). Hence there is a largest value of $q$, say $q^{\prime}$, for which the corresponding $R$-set is unbounded.

Define the $r$ integers $a, \cdots, l$ by

$$
a=q^{\prime} \alpha+q^{\prime}-1, \cdots, l=q^{\prime} \lambda+q^{\prime}-1 .
$$

Then the set $\left\{R_{a}^{B}, \ldots, l, i(r+1, \cdots, k ; n)\right\}$ is unbounded; and since when $h$ is any one of $a, \cdots, l$, and $\eta$ is the corresponding term in $\alpha, \cdots, \lambda$, we have

$$
q h+q-1=\left(q q^{\prime}\right) \eta+\left(q q^{\prime}\right)-1,
$$

we see that for each $q=2,3, \cdots$, set $\left\{R_{q a+q-1}^{B}, \cdots, q l+q-1, i(r+1, \cdots, k ; n)\right\}$ is bounded.

Now we are prepared to define the absolutely convergent series $\sum a_{(i)}$. Let

From the identity

$$
\begin{aligned}
a_{(a+1) t}, \cdots,(l+1) t, 0, \cdots, 0 & =(t+1)^{-2}, & t=1,2, \cdots ; \\
a_{(i)} & =0 & \text { otherwise. }
\end{aligned}
$$

$$
R_{(i)}^{C}=\sum a_{(i)} R_{(i-j)}^{B} \quad\left(0 \leqq j_{s} \leqq i_{s} ; s=1, \cdots, k\right)
$$

we then obtain the relation

$$
\begin{aligned}
R_{t a+t-1, \cdots, t l+t-1, i(r+1, \cdots, k ; n)}^{C} & =\sum_{s=1}^{t} \frac{1}{(t-s+1)^{2}} R_{s a+s-1, \cdots, s l+s-1, i(r+1, \cdots, k ; n) ;}^{B}
\end{aligned}
$$


and this permits us to conclude, as in the case $k=2$, that series $\sum c_{(i)}$ does not converge.

Theorems 1 and 2 combine to give the following theorem.

THEOREM 3. Let $B=\sum b_{(i)}$ be convergent. In order that the Cauchyproduct series $\sum c_{(i)}$ converge to the sum $C=A B$ for every absolutely convergent series $A=\sum a_{(i)}$, it is necessary and sufficient that the set $\left\{R_{(i)}^{B}\right\}$ be bounded.

We conclude with a remark on $\sigma$-convergent series. ${ }^{1}$ The property of being $\sigma$-convergent carries with it the boundedness of the set of all $\sigma$-sums (which sums correspond to the rectangular sums for Pringsheim convergence), and following the method of Theorem 1 the "Mertens theorem" can be established:

THEOREM 4. If $\sum a_{(i)}, \sum b_{(i)}$ are $\sigma$-convergent, one of them being absolutely convergent, then the Cauchy-product property holds: series $\sum c_{(i)}$ is $\sigma$-convergent to the sum $C=A B$.

The Pennsyluania State College

${ }^{1}$ For the definition and some properties of $\sigma$-convergence we refer to Convergence of multiply-infinite series, Amer. Math. Monthly vol. 52 (1945) pp. 365-376. 